\title{
Autosomal recessive cerebellar ataxia
}

INSERM

\section{Source}

INSERM. (1999). Orphanet: an online rare disease and orphan drug data base. Autosomal recessive cerebellar ataxia. ORPHA:1172

Autosomal recessive cerebellar ataxias (ARCA) are a heterogeneous group of rare neurological disorders involving both the central and peripheral nervous system (and in some cases other systems and organs), and characterized by degeneration or abnormal development of the cerebellum and spinal cord and, in most cases, early onset occurring before the age of 20 years. 